\title{
Beneficios de la vinculación Universidad-Sector Productivo
}

\author{
T.B. Pavón-Silva ${ }^{1}$, C. Barrera-Díaz ${ }^{1}$, V.F. Pacheco-Salazar ${ }^{1}$, J.C. Sánchez-Meza ${ }^{1}$, \\ G. Gómez-Beltran ${ }^{3}$ y R. Moreno-Colín ${ }^{4}$ \\ ${ }^{1}$ Facultad de Química. Universidad Autónoma del Estado de México, ${ }^{2}$ Instituto Tecnológico de Toluca, \\ ${ }^{3}$ Universidad Tecnológica del Valle de Toluca, "Fábrica de Galletas "La Moderna". \\ E-mail: th_pavon@yahoo.com.mx
}

(Recibido: agosto de 2005; aceptado: agosto de 2006)

\section{Resumen}

En 1999, una indu stria dedicada a la elaboración de alimentos y la Facultad de Química de la Universidad Autónoma del Estado de México (UAEMex) firmaron un convenio para realizar trabajos en la planta de tratamiento de aguas residuales (PTAR) de dicha empresa. Gracias a este convenio, personal de la empresa y alumnos, inicialmente de la Facultad de Química y posteriormente del Instituto Tecnológico de Toluca (ITT) y de la Universidad Tecnológica del Valle de Toluca (UTVT) de las carreras de Ingeniería Química, Química y Técnico Superior Universitario en Tecnología Ambiental, asesorados por catedráticos de la UAEMex, lograron mejorar las condiciones de control y operación de la PTAR; además de establecer diseños experimentales enfocados a investigaciones en campo que incrementaron el aprendizaje de alumnos y profesores. Este trabajo en equipo, dio como resultado la relación entre los profesores de estos centros educativos con el sector indus trial, así como la formación de recursos humanos en el área ambiental.

Descriptores: Relación Universidad-Empresa, formación de recursos humanos, gestión ambiental, educación supe rior.

\begin{abstract}
In 1999, an indus try ded icated to the food elab ora tion, and the Fac ulty of Chem is try of the Universidad Autónoma del Estado de México (UAEMex) signed an agree ment to carry out works in the wastewater tratment plant (WWTP) of this com pany. Thanks to this agree ment, per son nel of the com pany and stu dents, ini tially of the Fac ulty of Chem is try and later of the Instituto Tecnologico de Toluca (ITT) y de la Universidad Tecnológica del Valle de Toluca (UTVT) spe cif i cally in the fields of Chemical, Chemical Engineering and Technician in Environ men tal Engineering, ad vised by uni ver sity profes sors of the UAEMex, man aged to im prove the con ditions of con trol and op er a tion of the WWTP; be sides to es tablish fo cused ex per imental de signs to re search in field, and to in crease the learn ing of stu dents and pro fessors. This work in equip ment has pro moted the incensement in the re la tion ship of profes sors of these ed $u$ ca tional cen ters and the in dus try in the hu man re sources for mation in the en viron men tal area.
\end{abstract}

Keywords:Relation ship University-Industry, hu man resourcesfor mation, environ men talmanagement, highereducation. 


\section{Introducción}

La UAEMex tiene entre sus funciones sustantivas, la formación de recursos humanos y la difusión de su quehacer de investigación, por ello, es de suma importancia crear vínculos y dar respuesta a los problemas de los diferentes sectores con la aplicación del conocimiento que se genera desde universidades e institutos. (Viramontes, 2005).

Las instituciones de educación superior realizan cambios en la curricula, actualizando programas que se basan en las llamadas competencias, modernizando y flexibilizando esquemas de organización y centrando las actividades de enseñanza aprendizaje en el estudiante. Por ello, cuentan con mecanismos que favorecen la movilidad e intercambio tanto de alumnos como de maestros, diseña esquemas de vinculación $y$, en general, se enfoca a la transmisión y generación de conocimientos aplicados que resuelvan problemas y generen fuentes de empleo. En este contexto, la proyección de las Dependencias de Educación Superior (DES) se refleja en esquemas del servicio social y estancia profesional, jugando un papel de suma importancia, ya que con esto se cubre la necesidad de contacto con el mercado laboral de acuerdo con su perfil de egreso (Mendoza, 2005).

\section{Desarrollo}

El problema inicial dentro de la empresa con la que se firmó el convenio de colaboración, era el inadecuado funcionamiento del sistema de tratamiento de aguas residuales y la imposibilidad de no contar con un especialista para el control del proceso; por consiguiente, se maduró la idea de que la Universidad Autónoma del Estado de México a través de la Facultad de Química, estableciera un programa de asesoría para el control y manejo del proceso.

Como antecedente, la empresa ya tenía relación con el personal del laboratorio acreditado de servicios externos de la Facultad de
Química, a través de la venta de los análisis fisicoquímicos sobre la calidad de agua tratada, que en ese momento contaba con una concesión de CNA para determinaciones analíticas, evaluando parámetros como $\mathrm{pH}$, conductividad, sólidos suspendidos totales, sólidos volátiles totales, sólidos totales, detergentes, grasas y aceites, DQO y DBO. En ese momento, se contaba con porcentajes de remoción de 93, 90.2, y 57.25 para DBO, DQO y ST; sin embargo, se observó el incumplimiento de la normatividad vigente, por lo que se involucró a personal de la Universidad para realizar el diagnóstico y operación del proceso de tratamiento de agua.

En la primera fase de la resolución del problema, catedráticos de la Facultad de Química y personal del Departamento de Ingeniería de Planta de la empresa, fijaron como objetivo maximizar los recursos disponibles en cuanto a equipo y control de proceso con una mínima inversión; así como poner en marcha y mantener una operación estable, generando la calidad de agua que cumpliera con la normatividad vigente.

Para que esto se llevara a cabo, se incorporaron a la empresa estudiantes (3 durante un semestre) a quienes se les asignaron actividades específicas de medición de parámetros de control y operación de la planta. En el caso de requerir análisis específicos, se acordó con la empresa que fueran realizados por la Facultad, mientras la empresa absorbía el costo. El proyecto permitió, a través de las estancias profesionales o el servicio social de alumnos de las carreras de Ingeniería Química y Química, realizar diversos proyectos concernientes al área de tratamiento de agua residual industrial. Esto permitió a la empresa, avances importantes en cuanto a incentivos fiscales (condonación en precio de extracción de agua, depreciación acelerada, arancel cero) aunado al cumplimiento de protección al ambiente y la salud de la población.

Con el manejo adecuado de la planta, en corto tiempo se estableció el control en la 
operación del proceso. Se obtuvieron resultados favorables cambiando los difusores de aire del reactor biológico, mamparas de los clarificadores de alta tasa de remoción, equipo de control de $\mathrm{pH}$, necesarios en el control de proceso; así, en junio del 2000 la planta cumplió con los parámetros de la NOM-SEMARNAT-001, 1996, solicitándose los incentivos fiscales descritos arriba y recuperando parte de la inversión realizada.

Como todo proceso industrial de mejora y crecimiento continuo, la empresa ha evolucionado, creciendo tanto en líneas de producción de galletas, como en la incorporación de nuevas líneas. En una de ellas, la de pastas, en la cual su principal materia prima es la grasa líquida, razón por la cual las características de la calidad del agua de descarga nuevamente presentaron una variación, se procedió a una nueva caracterización y el establecimiento de un tren de tratamiento diferente al ya existente, éste consistió en la incorporación de una operación unitaria más, que fue la adición de un producto químico al inicio de la planta para la eliminación de grasas y aceites, así como de material particulado (Torres, 2005).

Para la realización de este trabajo fue necesario actualizar el diagnóstico de la calidad de agua por operación unitaria; encontrando los valores mostrados en la tabla 1, en donde se aprecia la evolución de los parámetros medidos y observando baja eficiencia de remoción de diversos parámetros. Se detectó una relación DBO/DQO de 0.19 a 0.56 , lo que indicó una calidad de agua muy variable a tratarse con un proceso biológico, explicando por qué el proceso no se mantenía controlado, ya que se encontraban elementos tóxicos para los microorganismos en el agua residual (Linares, 2004).

Con esta última fase, se implementó una operación unitaria más al inicio del proceso, así pues, ahora la planta de tratamiento de agua residual consta de las operaciones unitarias de coagulación-floculación, sedimentación primaria, cárcamo de bombeo, reactor anaerobio, reactor aerobio y desinfección con cloro. Actualmente, se lleva a cabo la evaluación del

Tabla 1. Caracterización del agua de acuerdo con diversas operaciones unitarias del sistema de tratamiento de agua

\begin{tabular}{|c|c|c|c|c|c|}
\hline Parámetro & Cribado & Cárcamo & $\begin{array}{c}\text { Reactor } \\
\text { Anaerobio }\end{array}$ & Rector Aerobio & Salida \\
\hline $\mathrm{DQO}\left(\mathrm{mg} \mathrm{L}^{-1}\right)$ & 3144.8 & 2389.8 & 1685.1 & 2316.7 & 1410.72 \\
\hline Acidez $\left(\mathrm{mg} \mathrm{L}^{-1}\right)$ & 61.4 & 118.1 & 190.3 & 78 & 58.3 \\
\hline Alcalinidad $\left(\mathrm{mg} \mathrm{L}^{-1}\right)$ & 193 & 261 & 371 & 398 & 395 \\
\hline $\mathrm{pH}$ & 7 & 6.7 & 5.9 & 7.3 & 7.3 \\
\hline Fósforo Total (mg L $\left.\mathrm{L}^{-1}\right)$ & 1.3 & 0.87 & 1.2 & 1.0 & 0.88 \\
\hline Nitrógeno Total (mg L $\left.{ }^{-1}\right)$ & 43 & 54.8 & 62.4 & N.D & 37.3 \\
\hline Nitratos $\left(\mathrm{mg} \mathrm{L}^{-1}\right)$ & 5.8 & 5.7 & 11.3 & 6.1 & 3.5 \\
\hline Nitritos $\left(\mathrm{mg} \mathrm{L}^{-1}\right)$ & 0.4 & 0.03 & 0.04 & 0.04 & 0.03 \\
\hline Sólidois Totales (mg L ${ }^{-1}$ ) & 1706 & 1500 & 1292 & 1658 & 1290 \\
\hline Sólidos volátiles (mg L ${ }^{-1}$ ) & 1133 & 927 & 602 & 909 & 437.3 \\
\hline Sólidos fijos (mg L ${ }^{-1}$ ) & 573 & 584 & 690 & 749 & 771.8 \\
\hline Sólidos suspendidos (mg L $\left.{ }^{-1}\right)$ & 464 & 163 & 116 & 520 & 74.1 \\
\hline Sólidos disueltos $\left(\mathrm{mg} \mathrm{L}^{-1}\right)$ & 1243 & 1348 & 1176 & 1138 & 1135 \\
\hline Grasas y Aceites $\left(\mathrm{mg} \mathrm{L}^{-1}\right)$ & 146.1 & N.D & 67.1 & N.D & 43.53 \\
\hline
\end{tabular}

N.D. No determinado 
sistema completo, pero como resultados previos se muestran las eficiencias de remoción para tres de los parámetros de una prueba realizada en planta, únicamente de la primera etapa, (Tabla 2).

Se observan eficiencias de remoción entre el 40 y el $87 \%$ para DQO, 42 y $92 \%$ para DBO y superiores al $94 \%$ para grasas y aceites, lo cual es importante, debido a que estas interfieren en la difusión del alimento y el oxigeno a los microorganismos del proceso biológico. Por otra parte, se observan relaciones de DBO/DQO de $0.27,0.65$ y 0.93 , esto indica una carga de materia orgánica adecuada (Metcalf y Eddy, 2003) para dar continuidad con el proceso biológico como tratamiento siguiente para cumplir con la normatividad del agua de descarga.

El interés de los estudiantes por realizar estancia en la empresa, ha permitido la incorporación de otras instituciones interesadas, estratificando el tipo y número de proyectos e incrementando la vinculación interinstitucional con la Universidad Tecnológica del Valle de Toluca (UTVT) y con el Instituto Tecnológico de Toluca (ITT).

El número total de alumnos que ha realizado algún trabajo de capacitación, investigación o desarrollo en la planta de tratamiento de aguas es de 46, mismo que ha servido para cumplir con el requisito curricular de estancia profesional o servicio social (un semestre). De éstos, algunos han continuado por un período adicional y tres han obtenido el título de técnico superior universitario; tres su titulación de ingeniero químico; dos como químicos; y otros se encuentran en proceso de titulación de Licenciatura, así también se generó una tesis de maestría en ciencia ambientales.

Con estos resultados, en un proceso de mejora continua y para contar con un mayor control, la empresa invirtió en material de pruebas rápidas para el pequeño laboratorio con el que cuenta el proceso de tratamiento de agua residual y así poder tomar decisiones rápidas en caso de presentarse un problema.

A los estudiantes, además de realizar actividades de control y operación del proceso de tratamiento de agua, se les asigna algún trabajo específico o proyecto de investigación, según sea el caso, en donde aplican los conocimientos adquiridos de su formación académica. Entre estos proyectos se pueden mencionar los siguientes:

- Diagnóstico integral del sistema de tratamiento de agua y de lodo residuales.

- Cambios y ajustes de operación en el tanque neutralizador: tiempo y velocidad de agitación y cambio de producto químico.

- Control de pH para dosificación automática (programación del equipo).

Tabla 2. Evaluación de la primera parte del tren de tratamiento en planta

\begin{tabular}{cccccccccc}
\hline & & Influente & \multicolumn{3}{c}{ Efluente con producto químico } & \multicolumn{3}{c}{ E (\%) } \\
\hline & DQO & DBO & GyA & DQO & DBO & GyA & DQO & DBO & GyA \\
\hline $\begin{array}{c}\text { Primera } \\
\text { Semana }\end{array}$ & 7489 & 3415 & N.D & 935 & 258 & N.D & 87.52 & 92.45 & N.D \\
$\begin{array}{c}\text { Segunda } \\
\text { Semana }\end{array}$ & 26602 & 6520 & 7946.2 & 3990 & 3750 & 16.22 & 85 & 42.48 & 99.8 \\
$\begin{array}{c}\text { Tercera } \\
\text { Semana } \\
\text { Cuarta }\end{array}$ & 3465 & 2670 & 2117.6 & 1283 & 835 & 26.16 & 62.97 & 68.73 & 98.76 \\
Semana & 4674 & 2163 & 327.12 & 2804 & 758 & 16.77 & 40.01 & 64.96 & 94.87 \\
\hline
\end{tabular}

N.D. No detrminado 
- Diagnóstico del proceso de aeración.

- Acondicionamiento del reactor anaerobio.

- Evaluación del proceso de sanitización en el área de producción.

- Prevención de necesidades por aumento de personal en la empresa.

- Implementación del tratamiento de lodo.

- Diseño del sistema de agitación para dosificación de productos químicos.

- Análisis de riesgos y determinación de puntos críticos de la PTAR.

- Re-diseño del sistema hidráulico y de bombeo.

- Determinación de coeficiente de transferencia de oxígeno.

- Establecimiento de un programa de mantenimiento preventivo.

- Identificación de la ocurrencia de microorganismos filamentosos (esponjamiento y formación de espuma).

- Determinación de la dosis óptima de cloro.

- Calidad empresarial.

- Estudios de carga orgánica (pruebas de biodegradabilidad del almidón) en un reactor piloto anaerobio.

- Modelación de lodos activados aplicando el Modelo ASM1.

Proyectos en proceso o que están por iniciarse:
- Relación entre DBO, DQO y COT.

- Estudios de respirometría para la determinación de factores cinéticos y parámetros fisicoquímicos en la modelación de lodos activados.

- Propuesta de implementación de reactores para determinar parámetros de modelación de lodos activados.

- Relación entre los factores fisicoquímicos y niveles de toxicidad.

- Re-uso de agua tratada dando cumplimiento a la NOM 003.

Actualmente, la empresa sigue admitiendo la estancia de estudiantes de las diferentes instituciones académicas. Cabe mencionar que la programación y control de proyectos se coordina en la Facultad de Química de la UAEMex, aunque siempre en colaboración con profesores-investigadores del ITT o de la UTVT y esto, a su vez, con el personal de la empresa.

Periódicamente, la empresa debe realizar análisis del agua de descarga, ya que los debe presentar ante el Municipio de Toluca y demostrar que está cumpliendo con los parámetros de calidad; esta es la manera en la que se da seguimiento al sistema de tratamiento. Además, el manejo de la calidad de agua de descarga es parte de un proceso de mejora continua en la empresa, ya que se ha certificado en varias de sus líneas de producción y el cuidado del ambiente es parte de esta certificación.

\section{Beneficios obtenidos}

En otro sentido, a nivel nacional se han establecido políticas en materia de ciencia y tecnología, a través del CONACYT, como la Ley de Ciencia y Tecnología aprobada por el $\mathrm{H}$. Congreso de la Unión en 2002; la creación de nuevos mecanismos de financiamiento como los fondos sectoriales (con secretarías de Estado 
Federales), y los fondos mixtos (con gobiernos Estatales y Municipales), entre otros, para apoyar la investigación científica y el desarrollo tecnológico.

En el fondo sectorial de SEMARNAT, en la convocatoria 2002, se solicitaron recursos para continuar con investigación aplicada, resultando beneficiada la Facultad para la adquisición de infraestructura y equipo, esto como resultado de la vinculación existente entre el sector productivo y la misma Facultad.

En la tabla 3, se indica de manera general y sintetizada, los beneficios obtenidos para los integrantes del Convenio de Colaboración Universidad - Sector Productivo.

Se ha cumplido específicamente con la formación de recursos humanos, tanto de la Facultad de Química de la UAEMex, como de la Universidad Tecnológica del Valle de Toluca y del Instituto Tecnológico de Toluca. La capacitación para el personal de la empresa es constante, a través de la suma de experiencias por el ejercicio de los proyectos y dinámica de trabajo entre estudiantes y profesores.

En materia de difusión de resultados, se ha reconocido al proyecto como caso de éxito en vinculación academia-empresa, otorgando a la empresa un reconocimiento por la Red Nacional de Consejos y Organismos Estatales de Ciencia y Tecnología en mayo de 2003. Asimismo, los profesores y alumnos involucrados en este proyecto, entre otros, han participado en los siguientes eventos académicos:

- XXXIX Congreso Interamericano de Ingeniería Sanitaria y Ambiental. 22-27 de agosto. San Juan de Puerto Rico.

- IX Congreso Nacional y III Congreso Internacional de Ciencias Ambientales. 12-14 de mayo de 2004 Huatulco, Oaxaca.

- Experiencias en gestión Ambiental en búsqueda de Vinculación IES - Empresa. IV Seminario Internacional CYTEDXVII. Un enfoque integrado para la gestión sustentable del agua. Experiencias en gestión y valoración del agua. 29-31 marzo del 2004. Universidad Nacional de Costa Rica.

- The First International Meeting on Environmental Biotechnology and Engineering. 6-8 september, 2004. México, D.F. ISBN 970-94112-0-9.

Con referencia a lo económico, la Facultad se ha beneficiado con el apoyo de recursos federales a través del proyecto de investigación titulado "Reingeniería de una planta de tratamiento de agua de una industria alimentaria".

Tabla 3. Beneficios obtenidos por los participantes

\section{Universidad}

Formación y capacitación de recursos humanos (servicio social, prácticas profesionales, tesis)

Experiencia para profesores

Difusión de resultados de congresos

Obtención de recursos de fondos sectoriales

Vinculación interistitucional

Interdisciplinariedad en problemas ambientales
Industria

Capacitación de recursos humanos de la empresa

Imagen empresaria como industria limpia Incentivos fiscales al dar cumplimiento a la normatividad

Beneficios económicos al optimizar los procesos y gasto de reactivos

Resolución de problemas específicos a menor costo Innovación y desarrollo tecnológico 


\section{Conclusiones}

Los profesores de la Facultad de Química, integrados en el Cuerpo Académico de Química Ambiental, promueven una vinculación más efectiva entre su quehacer científico y las necesidades de los sectores productivos, con trabajo interdisciplinario y procurando dar alternativas de solución a los diferentes problemas relacionados con el ambiente.

En la realización de los proyectos de investigación se busca:

- Oportunidad de crecimiento mutuo en todas las áreas específicas relacionadas con química y ambiente.

- Formar recursos humanos.

- Adquirir experiencia en la aplicación de conocimiento en solución de problemas específicos.

- Beneficios económicos para las partes, y sobre todo,

- Beneficio social.

La vinculación, a través de la Extensión Universitaria, relaciona los problemas específicos de los demandantes, obliga a la búsqueda de información y actualiza el conocimiento en la materia. Con creatividad y un ambiente propicio, permite incursionar en el proceso de la investigación con resultados y experiencias de la práctica. Asimismo, se lleva a las aulas para motivar el espíritu emprendedor de los alumnos con credibilidad, certeza, coherencia y congruencia.

Esta vinculación mantiene la actividad y la cataliza en el ámbito de las funciones sustantivas universitarias de manera interrelacionada; permite la creación de sinérgica al resolver un problema social; permite mejorar los procesos de enseñanza-aprendizaje y fomenta a los estudiantes a la investigación con creatividad y actitud emprendedora. Finalmente, crea confianza y credibilidad personal y social.

\section{Agradecimientos}

Se agradece el apoyo a las autoridades institucionales del sector educativo participante, a la Empresa y al apoyo del Fondo Sectorial CONACYT-SEMARNAT a través del proyecto "Reingeniería de una planta de tratamiento de aguas residuales de la industria alimentaria", con clave C01-0502.

\section{Referencias}

Linares-Hernández I. (Septiembre 2004). Diagnóstico de la operación de una planta de tratamiento de agua residual de la industria alimentaria. Tesis de Licenciatura en Química. Universidad Autónoma del Estado de México. Facultad de Química, Toluca, Estado de México.

Mendoza-Puga L. (2005). Vinculación, Universidad, Sector productivo y Gobierno. VIISeminario Nacional Territorio Industria y Tecnología. Memoria CD-Room.

Metcalf and Eddy (2003). Wastewater Engineering Treatment and Reuse. Four Edition, Mc Graw-Hill.

Torres-Blancas T. (2005). Comparación de procesos fisicoquímicos para la eliminación de COT en agua residual indus trial. Tesis de Licenciatura en Química, Universidad Autónoma del Estado de México, Facultad de Química, Toluca, Estado de México.

Viramontes-Brown R. (2005). Acciones para lograr que la innovación y el desarrollo tecnológico impulsen el crecimiento de la economía y la productividad de la Industria. Innovación y competitividad, No. 18, Abril.

\section{Bibliografía sugerida}

Enkerlin E., Cano G., Garza R. y Vogel E. (1997). Ciencia ambiental y desarrollo sostenible. International Thomson Editores, México. 
Instituto Nacional de Ecología (1999). Gestión ambiental. http://www.ine.gob.mx.

Millán J. y Concheiro A.A. (2000). México 2030. Nuevo siglo, nuevo país. Fondo de Cultura Económica, México.

Rocher M. and Roux G. (2001). Excess Sludge Reduction in Activated Sludge Processes by Integrating Biomass Alka line Treatment Wat. Sci.Tech., Vol. 44, No.2-3, pp.437-444.

Pavón-Silva T., Linares-Hernández I., Pacheco-Salazar V., Barreda-Díaz C., Colín-Cruz A. (2004). The First Internacional Meeting on Environmental and Biotechnology and Engineering. Diagnóstico de la operación de una planta de aguas residuales de industria alimentaria, September 6-8, México.
Pavón-Silva T.B., Pacheco-Salazar V.F., Guillermo-Muñoz R., Pérez-López I. y Moreno-Colín R. (2002). II. Encuentro Institucional de Vinculación Universitaria, Universidad Autónoma del Estado de México. Vinculación en el área ambiental empresa-institución de educación supe rior.

Pacheco-Salazar V.F. (2000). Curso de legislación ambiental. Maestría en Ciencias Ambientales. Facultad de Química, U.A.E.M.

Villalever L. y Flores-Crespo (2002). Las universidades tecnológicas mexicanas en el espejo de los institutos universitarios de tecnología francés. En: Revista Mexicana de Investigación Educativa, Vol. 7, No. 14, México, DF. 


\section{Semblanza de los autores}

Thelma Beatriz Pavón-Silva. Es licenciada en Química por la Universidad Nacional Autónoma de México, UNAM, maestra en ingeniería ambiental por la Facultad de Ingeniería de la UNAM y doctora en ingeniería por la Universidad Autónoma del Estado de México (UAEMex) en el Centro Interamericano de Recursos del Agua. Ha laborado como docente desde el año 2002, impartiendo cursos de nivel licenciatura y posgrado; asimismo, cuenta con publicaciones en el ámbito nacional e internacional y ha participado en congresos. Actualmente pertenece al Sistema Nacional de Investigadores.

Carlos Eduardo Barrera-Díaz. Obtuvo la licenciatura en ingeniería química en la Facultad de Química, UAEM, asimismo, la maestría en ingeniería ambiental por la Universidad de British Columbia, Canada, y el doctorado en ingeniería ambiental por la Universidad Autónoma Metropolitana. Ha impartido docencia a nivel licenciatura y posgrado, actualmente es Coordinador de Investigación y Posgrado de la Facultad de Química, UAEM. Cuenta con publicaciones de nivel nacional e internacional y participación en congresos. Tiene el reconocimiento de Profesor Perfil Promep, otorgado por la Secretaría de Educación Pública y Pertenece al Sistema Nacional de Investigadores.

Víctor Francisco Pacheco-Salazar. Es ingeniero químico y maestro en ingeniería química por la UNAM, asimismo, es doctor en ingeniería (ITT, 2004-2006). Fue investigador del SNl de 1986-a 1990 y laboró en el IIB-UNAM, participando en el desarrollo biotecnológico (Biofermel) licenciado comercialmente. Desde 1982, realiza actividades docentes e investigación en la FQ-UAEM. Ha impartido más de 100 conferencias y ha publicado en revistas arbitradas, así como de divulgación. Sus líneas de interés son la vinculación academia-empresa en reingeniería de plantas de tratamiento, optimización de procesos y sistemas de calidad. Perteneció a la administración universitaria de la FQ-UAEM como director, coordinador de posgrado y de vinculación. En la UAEM fue coordinador general de investigación. Con referencia a la administración pública del Estado de México fue director general en la SE-GEM (1993-1994); asesor SECyBS (1994-1997) y director de desarrollo tecnológico, COMECYT (2001-2003).

Juan Carlos Sánchez-Meza. Es químico farmacéutico biólogo con maestría en ciencias, en la especialidad de toxicología, realizada en el CINVESTAV-IPN. Labora como profesor-investigador en la Facultad de Química, Universidad Autónoma del Estado de México. Imparte los cursos de toxicología ambiental y evaluación del riesgo en la licenciatura de químico farmacéutico biólogo, así como en el posgrado en Ciencias Ambientales de la UAEMex. La prin cipal área de interés en investigación está relacionada con la evaluación de riesgos a la salud y al ambiente, derivados de la exposición a agentes tóxicos. Actualmente se encuentra realizando estudios de evaluación de la toxicidad de aguas residuales industriales a través de bioensayos.

Guillermina Gómez-Beltrán. Tiene la licenciatura en biología y el grado de maestra en ciencias de agua por la Universidad Autónoma del Estado de México. Es profesora investigadora de la Universidad Tecnológica del Valle de Toluca e imparte asignaturas en la carrera de técnico supe rior universitario en tecnología ambiental. Ha participado en el desarrollo de trabajos aplicados a diferentes giros industriales enfocados con el área ambiental.

Rodrigo Moreno-Colín. Ingeniero electricista por la Universidad Michoacana, desarrolló trabajos en indu stria azucarera, cuenta con amplia experiencia en el área de mantenimiento de maquinaria y equipo. Ha recibido capacitación en Brasil, Europa y Estados Unidos. Apoya a los estudiantes para su desarrollo personal y de capacitación dentro del sector empresarial. Actualmente se desarrolla como gerente de ingeniería de planta de la empresa: Fábrica de Galletas "La Moderna”. 